J. Phys. IV France 138 (2006) 181-190

(C) EDP Sciences, Les Ulis

DOI: $10.1051 / \mathrm{jp} 4: 2006138021$

\title{
Élaboration de films de molécules organiques par ablation par laser UV
}

\author{
M.A. Hernandez-Perez ${ }^{1}$, C. Garapon ${ }^{1}$, C. Champeaux ${ }^{2}$, A.W. Coleman ${ }^{3}$ \\ et L. Guy ${ }^{4}$ \\ ${ }^{1}$ Laboratoire de Physico-Chimie des Matériaux Luminescents, UMR 5620 CNRS, \\ Université Lyon-I, 10 rue André-Marie Ampère, 69622 Villeurbanne Cedex, France \\ 2 Science des Procédés Céramiques et Traitement de Surface, UMR 6638 CNRS, \\ Université Limoges, 123 Av. Albert Thomas, 87060 Limoges Cedex, France \\ ${ }^{3}$ Institut de Biologie et Chimie des Protéines, UPR 412 CNRS, 7 passage du Vercors, \\ 69367 Lyon Cedex 07, France \\ ${ }^{4}$ Laboratoire de Chimie, UMR 5182 CNRS, École Normale Supérieure de Lyon, \\ 46 allée d'Italie, 69364 Lyon Cedex 07, France
}

\begin{abstract}
Résumé. Les potentialités des méthodes de dépôt par ablation laser (PLD) pour la préparation de films minces de matériaux organiques sont illustrées par un bref rappel bibliographique et par des résultats expérimentaux concernant des molécules d'intérêt biologique (acides aminés, calix-arènes, protéines). Les films sont préparés par PLD avec un laser KrF sans dégradation de la structure chimique des molécules dans une gamme de fluences de quelques dizaines à quelques centaines de $\mathrm{mJ} / \mathrm{cm}^{2}$. Les propriétés structurales et optiques des films sont étudiées en fonction de la fluence du laser et mettent en évidence des arrangements moléculaires particuliers induits par cette méthode de dépôt. Le guidage optique a été obtenu pour des films de toutes ces molécules.
\end{abstract}

\section{INTRODUCTION}

L'une des applications actuelles des lasers UV est la réalisation de films minces, dont les propriétés, qu'elles soient optiques, magnétiques, mécaniques, électroniques, etc..., sont susceptibles d'être étudiées d'un point de vue fondamental ou de donner lieu à des applications nouvelles. L'absorption du faisceau d'un laser UV pulsé à la surface d'une cible du matériau à déposer se traduit par une éjection de matière, qui se dépose sur un substrat placé à quelques $\mathrm{cm}$ de la cible. Cette méthode, particulièrement performante pour les oxydes complexes, est applicable à toutes sortes de matériaux (métaux, carbone, semi-conducteurs ...). Elle permet le contrôle de la composition chimique, de la phase cristalline et de la texture grâce au choix des paramètres (fluence, température, substrat) et à la possibilité d'effectuer les dépôts en présence d'un gaz réactif ou non. Elle permet également d'ajuster l'épaisseur déposée de manière précise et d'effectuer aisément des dépôts successifs de matériaux différents [1].

L'utilisation de cette méthode pour le dépôt de films organiques voire biologiques est beaucoup plus récente et constitue une alternative aux dépôts par évaporation thermique et à partir de solutions, qui sont difficilement applicables pour certains matériaux [2]. Or les propriétés électroniques et optiques des films organiques peuvent conduire à des applications extrêmement importantes, par exemple dans le domaine des diodes électro-luminescentes [3], des capteurs chimiques et biochimiques [4] ou du stockage d'informations [5]. Ces films peuvent également permettre d'étudier des propriétés d'autoassemblage et de reconnaissance moléculaire d'un grand intérêt d'un point de vue fondamental [6]. Sont concernés aussi bien de petites molécules que des polymères de poids moléculaire élevé et même des macromolécules complexes comme les protéines. Deux méthodes, basées sur l'ablation par un laser pulsé UV sont utilisées: soit le dépôt par laser pulsé conventionnel (PLD) soit l'évaporation laser assistée par la matrice (MAPLE) [2]. Cette dernière méthode consiste à disperser les molécules à déposer dans 
une matrice gelée constituée de petites molécules volatiles qui absorbent préférentiellement le faisceau laser UV. Ces molécules sont évaporées et éliminées par pompage et permettent par entraînement la désorption des plus grosses molécules sans dégradation. Chacune de ces deux méthodes présente des avantages et des inconvénients, selon l'objectif à atteindre. La méthode MAPLE est généralement considérée comme susceptible d'améliorer la conservation des molécules, en particulier des protéines. La PLD a néanmoins permis le dépôt de films de molécules organiques de taille et de complexité diverses sans dégradation.

Le dépôt de molécules organiques soulève par ailleurs de nouvelles questions sur les mécanismes d'ablation. En effet, les études antérieures ont longtemps porté essentiellement sur l'ablation des polymères, avec pour but la structuration d'un matériau massif plutôt que la réalisation de films [7]. Elles doivent donc être complétées dans la mesure où l'intérêt se porte sur la conservation des molécules ablatées. De plus, deux types de molécules sont à considérer. Dans le cas du dépôt de polymères, l'ablation se traduit par une dépolymérisation et la re-polymérisation sur le substrat de monomères ou de fractions de chaînes [8]. Au contraire les molécules, dont l'organisation en phase solide ne comporte pas d'enchevêtrements, peuvent être ablatées et transférées sans décomposition.

Notre objectif ici est de rappeler brièvement quelques faits expérimentaux dans le domaine du dépôt de molécules organiques par dépôt par laser pulsé conventionnel ainsi que par MAPLE, sans aborder le cas des polymères. Des données bibliographiques sur l'ablation des matériaux organiques peuvent être trouvées dans [9]. Nos propres résultats expérimentaux [10], concernant le dépôt, par ablation laser conventionnelle, de films de molécules organiques d'intérêt biologique, acides aminés [11], calixarènes [12] et protéines [13] et l'étude de leurs propriétés structurales et optiques, seront ensuite présentés.

\section{DÉPÔT DE FILMS ORGANIQUES PAR ABLATION LASER}

\subsection{Dépôt par laser pulsé conventionnel}

La préparation de films organiques, qui s'est développée à partir des années 90, concerne essentiellement des molécules semi-conductrices comme le pentacène $[14,15]$ ou luminescentes, comme le tris-8hydroxyquinoline d'aluminium (Alq3) ou le complexe cuivre- phtalocyanine (CuPc) [16, 17] pour des applications dans le domaine de l'électronique, de l'optique non linéaire et des diodes électroluminescentes organiques (OLED). Ces films sont préparés grâce à des lasers UV de longueurs d'onde s'étendant de $248 \mathrm{~nm}$ à $355 \mathrm{~nm}$ avec des fluences de l'ordre de quelques dizaines à quelques centaines de $\mathrm{mJ} / \mathrm{cm}^{2}$, généralement sous vide. Par exemple, des films de pentacène, dont la structure cristalline et l'orientation préférentielle ont pu être contrôlées grâce à la température du substrat et la fluence, ont été obtenus avec des propriétés de conductivité similaires voire meilleures que celles de films préparés par évaporation thermique. Les premiers films de protéines, fibroine de la soie [18]. kératine et collagène [19] ont été réalisés par Tsuboi à la fin des années 90, sans destruction de la structure primaire mais une modification partielle de la conformation [20],

\subsection{Dépôt assisté par une matrice : MAPLE}

Parallèlement au développement de la PLD de molécules organiques, la méthode MAPLE a été mise au point par Chrisey et al [2]. Cette méthode a été utilisée aussi bien pour déposer des petites molécules comme l'anthracène [21] que des polymères, comme le polythiophène [22]. Mais son utilisation principale reste le dépôt de grosses molécules biologiques comme les protéines [23, 24]. Elle a pu être mise en œuvre avec des lasers de plus courte longueur d'onde comme ArF, qui induisent une décomposition des molécules organiques pour la PLD conventionnelle., La gamme des fluences utilisées est similaire, de quelques dizaines à quelques centaines de $\mathrm{mJ} / \mathrm{cm}^{2}$. La liste des solvants utilisés est variée et fait apparaître que le critère de l'absorption privilégiée par les molécules du solvant 
nécessiterait d'être précisé. En effet, cette méthode s'applique pour des solvants non absorbants (tels que l'eau) ou des molécules absorbantes (telles que certaines protéines) [25]. Cette méthode, qui a pour but de minimiser les processus de dégradation moléculaire, présente effectivement l'avantage de préserver la structure des protéines puisque la conservation de l'activité biologique a été mise en évidence [23, 24]. Elle présente également l'avantage de ne nécessiter qu'une faible quantité des molécules à déposer, souvent onéreuses ou rares, grâce à la dilution, de l'ordre de $1 \%$. Elle a néanmoins des inconvénients, comme le faible taux de dépôt et la rugosité importante des films ou comme des conditions expérimentales délicates à mettre en œuvre à cause de la nécessité d'employer un porte-cible refroidi et à la difficulté du choix du solvant approprié à chaque molécule.

On peut ajouter que de nouvelles méthodes, dérivées du MAPLE, [2] sont apparues ensuite, par exemple, la méthode MAPLE-Direct Write, qui consiste à transférer sous l'action d'un laser des biomolécules ou même des cellules vivantes, diluées dans une matrice, d'une cible comportant une série de molécules différentes vers un substrat situé à quelques $\mu \mathrm{m}$ selon un processus de type «machine à écrire ». Une autre variante "Resonant Infrared-PLD ou MAPLE" consiste à effectuer la désorption des molécules grâce à un laser IR excitant sélectivement une vibration des molécules.

\subsection{Analyse des mécanismes et simulation}

De très nombreuses études ont été consacrées à la détermination du rôle des paramètres expérimentaux intervenant dans la méthode MALDI [26] (Désorption/ionisation par laser assistées par matrice), méthode utilisée pour l'analyse des protéines dont est dérivée le MAPLE. Ce n'est le cas ni pour la PLD, ni pour le MAPLE. De plus des renseignements, portant sur le déroulement de l'ablation et le rôle de certains facteurs expérimentaux, ont été apportés récemment par la simulation numérique, en particulier par Zhigilei et al [27]. Bien que, pour ces simulations, les paramètres utilisés correspondent au cas du MALDI, les phénomènes qu'elles font apparaître sont tout à fait transposables au cas du dépôt par PLD. Les modèles proposés considèrent généralement que l'ablation intervient lorsque la densité d'énergie absorbée dépasse un seuil lié à l'énergie de cohésion du matériau. Ces simulations font apparaître en particulier, l'existence d'un passage entre un régime de désorption à basse fluence à un régime d'ablation collective à haute fluence. En ce qui concerne les effets photochimiques provoquant la rupture de liaisons covalentes, ils ont été clairement mis en évidence expérimentalement et modélisés ensuite dans le seul cas de l'ablation des polymères [28] et commencent à être introduits dans les modélisations de l'ablation de plus petites molécules.

\section{FILMS DE MOLÉCULES D'INTÉRÊT BIOLOGIQUE}

Nous avons étudié les potentialités de la méthode de dépôt par ablation laser UV pour la réalisation de films organiques en choisissant trois types de molécules, de taille et de complexité croissante et susceptibles d'applications dans le domaine des capteurs chimiques ou biochimiques par leurs propriétés optiques ou physico-chimiques. Il s'agit de deux acides aminés, la phénylalanine (Phe) et la tyrosine (Tyr), dont les propriétés de fluorescence et de chiralité sont importantes pour l'analyse de protéines, d'un calix-[4]-arène substitué, macrocycle possédant des propriétés de complexation sélective d'ions et de molécules, dont des gaz ou des protéines, et enfin de deux protéines globulaires, l'albumine sérique bovine et l'hémoglobine [10]. Nous résumons ici les résultats les plus représentatifs des particularités induites par cette méthode.

\subsection{Conditions expérimentales de préparation des films}

Les films sont réalisés, sur des substrats de silice ou de verre, à température ambiante par ablation par un laser $\operatorname{KrF}(\lambda=248 \mathrm{~nm})$ de cibles constituées de poudres compactées. Les dépôts sont effectués sous pompage dynamique, la pression de base de $10^{-8}$ mbar augmentant jusqu' à $10^{-6}$ à $10^{-5}$ mbar selon la 
fluence par suite de la mise en phase vapeur des molécules. La fluence est ajustée par un atténuateur de faisceau dans une gamme de quelques dizaines de $\mathrm{mJ} / \mathrm{cm}^{2}$ à environ $1 \mathrm{~J} / \mathrm{cm}^{2}$ pour un spot de $2 \mathrm{~mm}^{2}$, dont la taille a été déterminée par l'impact sur des couches de PMMA d'une impulsion unique d'énergie $25 \mathrm{~mJ}$. Trois montages expérimentaux ont été utilisés, se différentiant essentiellement par le laser utilisé (Lambda Physik EMG 101 ou 103 en résonateur stable ou LPX 100 en résonateur instable). Les résultats sont tout à fait similaires, si on tient compte des différences entre les valeurs absolues des fluences et entre les répartitions énergétiques des faisceaux. Il faut d'ailleurs remarquer qu'il est très difficile de comparer les résultats publiés du point de vue de la fluence, à cause des différentes procédures, rarement explicitées, grâce auxquelles elle est déterminée.

\subsection{Propriétés de films d'acides aminés}

La tyrosine Tyr dérive de la phénylalanine Phe $\left(\mathrm{C}_{6} \mathrm{H}_{5}-\mathrm{CH}_{2}-\mathrm{CH}\left(\mathrm{NH}_{2}\right)-\mathrm{COOH}\right)$ par l'addition d'un groupe $\mathrm{OH}$ en para sur le cycle benzénique, ce qui modifie sensiblement les interactions moléculaires. Les films de quelques centaines de nm d'épaisseur, déposés dans une gamme de fluences s'étendant de 40 à $700 \mathrm{~mJ} / \mathrm{cm}^{2}$, sont transparents et sans gouttelettes de taille micrométrique.

\subsubsection{Conservation de la structure chimique}

La conservation de la structure chimique des molécules a été vérifiée par FTIR en configuration ATR (réflexion totale atténuée). La figure 1a présente les spectres IR de certains films de Phe représentatifs, dont la comparaison avec celui de la poudre de phénylalanine montre qu'ils comportent toutes les bandes de vibration correspondant à la forme zwitterionique de la molécule [10]. La conservation de la structure chimique de la molécule est donc vérifiée.

Cependant, des différences existent entre les films et la poudre. Certaines raies des spectres des films sont décalées par rapport à leur position pour la poudre: de 1558 à $1588 \mathrm{~cm}^{-1}$ et 1410 à $1403 \mathrm{~cm}^{-1}$ pour les vibrations d'élongation asymétrique et symétrique du groupe $\mathrm{COO}^{-}$, de 1495 à $1515 \mathrm{~cm}^{-1}$ pour la déformation symétrique de $\mathrm{NH}_{3}^{+}$et de $3000 \mathrm{~cm}^{-1}$ à $2900 \mathrm{~cm}^{-1}$ pour la vibration d'élongation de $\mathrm{NH}_{3}^{+}$. Par comparaison avec les spectres de molécules isolées libres de toutes liaisons $\mathrm{H}$ et ceux de molécules en solution aqueuse soumises à de fortes liaisons $\mathrm{H}$, nous avons attribué ces déplacements à un renforcement des liaisons hydrogène dans les films par rapport à la poudre [10]. Nous pouvons donc conclure que, si la structure chimique des molécules est préservée, leur arrangement est légèrement modifié lors du dépôt par laser.
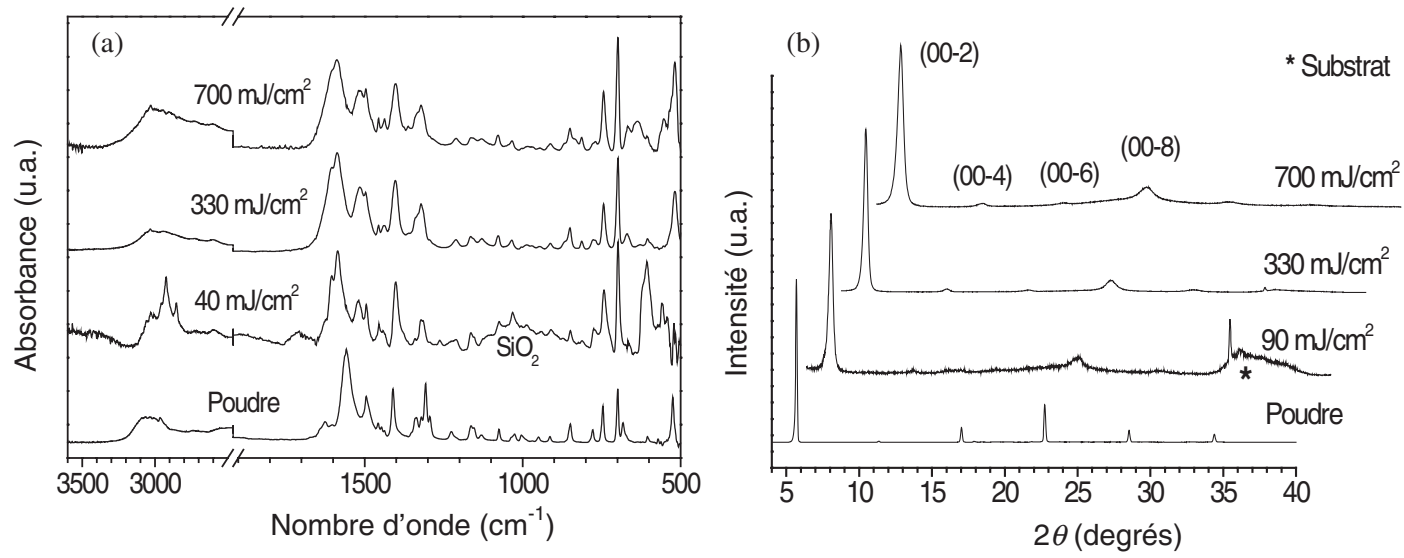

Figure 1. Spectres IR (a) et de diffraction X (b) de la poudre et de films de Phe déposés à différentes fluences. 
Un deuxième point concerne les films déposés à basse fluence. Pour le film déposé à $40 \mathrm{~mJ} / \mathrm{cm}^{2}$, en plus des bandes de la phénylalanine, le spectre comporte des bandes de vibration du groupe $\mathrm{CH}_{2}$ vers $2900 \mathrm{~cm}^{-1}$ d'intensité notablement plus élevée que pour les films déposés à plus haute fluence et des bandes peu intenses vers $1700 \mathrm{~cm}^{-1}$ et à $610 \mathrm{~cm}^{-1}$ d'origine inconnue. La présence de ces bandes additionnelles indique qu'une décomposition partielle de la molécule a lieu lors des dépôts à basse fluence. Cette observation de l'existence d'une fluence seuil pour la conservation de l'intégrité de la molécule semble indiquer que deux mécanismes différents sont en œuvre selon la fluence. Par analogie avec la description issue des simulations [27], nous supposons qu'à basse fluence existe un régime de désorption, molécule par molécule, qui implique la décomposition d'un certain nombre de molécules, régime qui laisse la place à plus haute fluence à un régime d'ablation, collectif, dans lequel la libération d'agrégats protège l'intégrité des molécules.

En ce qui concerne la tyrosine, la similitude entre les spectres IR de la poudre et des films montre que l'intégrité de la molécule est conservée, ceci dans la même gamme de fluences que pour la phénylalanine. On note toutefois un élargissement des bandes de vibration du cycle phénolique et une modification d'intensité des bandes de vibration du groupe $\mathrm{OH}$, qui peuvent être attribuées, comme pour la phénylalanine, à une organisation modifiée par rapport à la poudre, en l'occurrence un arrangement plus désordonné [11].

\subsubsection{Propriétés structurales}

Les spectres de diffraction des rayons $\mathrm{X}$ des films déposés à différentes fluences ont été comparés à ceux de la poudre pour les deux acides aminés. Dans le cas de Phe (Figure 1 b) ils montrent que, quelle que soit la fluence de dépôt, les films sont cristallisés dans la même phase monoclinique que la poudre et sont très fortement orientés. L'absence de raies de diffraction sur les spectres des films de Tyr, contrairement au cas de la poudre, montre que ces films sont amorphes, ce qui confirme l'attribution de l'élargissement des bandes de vibration IR à un environnement désordonné. La structure cristalline de Phe est caractérisée par une alternance de doubles couches, regroupant les cycles benzéniques d'une part et les groupes acides et amines d'autre part. Cette structure, induite par les liaisons hydrogène entre les groupes $\mathrm{COO}^{-}$et $\mathrm{NH}_{3}^{+}$, favorise le renforcement des liaisons $\mathrm{H}$ mis en évidence par IR [10]. La cristallisation de Tyr, qui est d'ailleurs mal connue à l'état massif, est rendue plus difficile que pour Phe, à cause de la présence du substituant $\mathrm{OH}$ sur le cycle, qui rend impossible la structure en doubles couches.

La modification de l'environnement local des molécules est métastable, il relaxe à l'échelle de quelques mois voire années pour Phe et plus rapidement, quelques jours, pour Tyr, comme l'atteste l'évolution des spectres IR avec le temps [11]. Ce comportement peut s'expliquer par les caractéristiques de la PLD (espèces de forte énergie cinétique, taux de dépôt instantané élevé favorisant les structures métastables).

\subsubsection{Propriétés optiques: indices de réfraction}

Les indices de réfraction des films de Phe et Tyr ont été mesurés pour plusieurs longueurs d'onde par spectroscopie des lignes noires. La figure 2 représente la variation des indices avec la fluence, pour les polarisations TE (transverse électrique) et TM (transverse magnétique) de films de Phe et Tyr. Les indices des films de Phe sont indépendants de la fluence et fortement anisotropes, ce qui est en bon accord avec la structure cristallisée et fortement orientée des films. Les indices des films de Tyr sont isotropes et augmentent avec la fluence, ce qui indique une densification de la structure amorphe des films, lorsque l'énergie des espèces déposées augmente.

De la mesure des épaisseurs, il est possible de remonter au taux de dépôt, qui suit une loi semilogarithmique en fonction de la fluence et qui permet de déterminer une fluence seuil de l'ordre de $80 \mathrm{~mJ} / \mathrm{cm}^{2}$ pour la phénylalanine. 

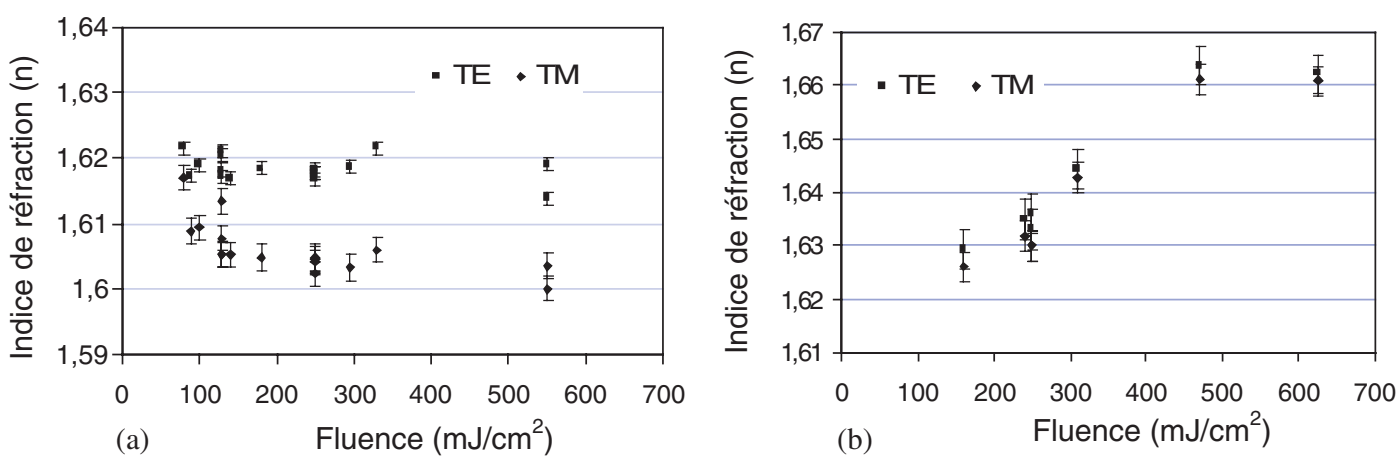

Figure 2. Indices de réfraction à $543,5 \mathrm{~nm}$ des films de Phe (a) et Tyr (b) en fonction de la fluence.

\subsubsection{Propriétés optiques au niveau moléculaire}

Les spectres d'absorption et de fluorescence (Figures 3 et 4) ont confirmé la conservation de la structure moléculaire, par l'observation des raies caractéristiques des transitions entre le singulet fondamental $\mathrm{S}_{0}$ et le premier singulet excité S1 pour chacune des molécules.
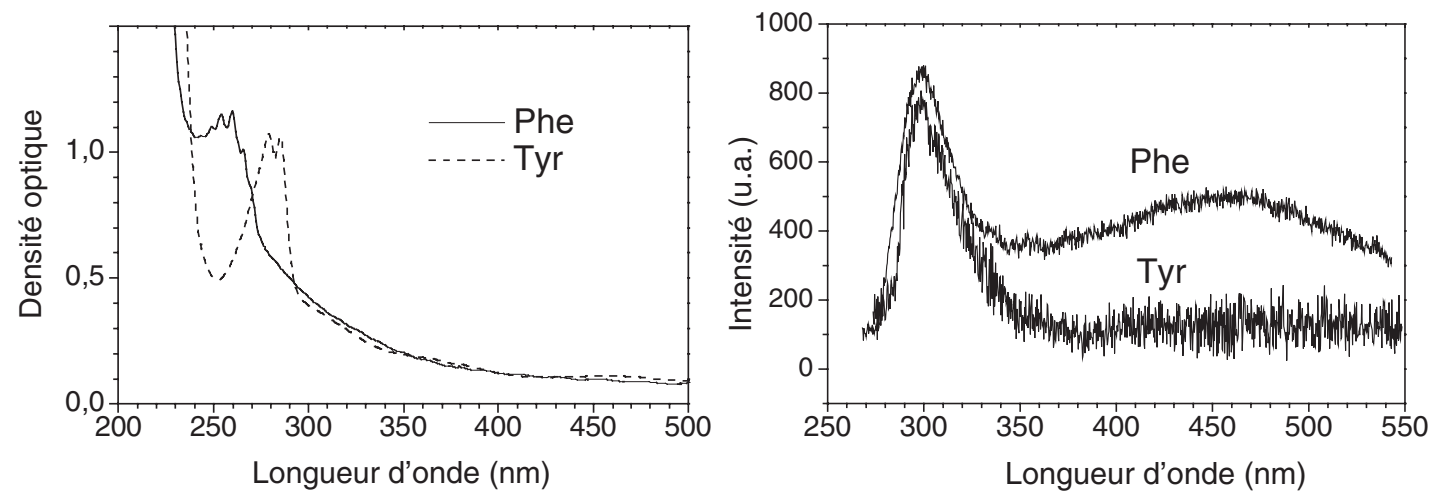

Figure 3. Spectres d'absorption de films de Phe et Tyr de Figure 4. Spectres de fluorescence de films de Phe et 2,6 et $0,42 \mu \mathrm{m}$ d'épaisseur déposés à 250 et $80 \mathrm{~mJ} / \mathrm{cm}^{2}$. Tyr déposés à $130 \mathrm{et} 190 \mathrm{~mJ} / \mathrm{cm}^{2}$. $\left(\lambda_{\text {exc }}=230 \mathrm{~nm}\right)$.

Ces mesures ont également mis en évidence l'intervention de processus photochimiques, de nature différente pour Phe et Tyr. Lorsque la fluence de dépôt augmente, une légère coloration jaune des films se développe et se traduit par une bande d'absorption large, qui s'étend de 200 à $400 \mathrm{~nm}$ et dont la contribution est plus marquée pour Tyr. Dans le cas de Phe, cette bande, qui apparaît également lorsque les films sont soumis à une irradiation UV vers $250 \mathrm{~nm}$, est responsable d'une émission centrée vers $470 \mathrm{~nm}$. Nous avons attribué ces bandes à des molécules de Phe, entre lesquelles existerait une interaction similaire à celle qui est à l'origine des bandes d'excimères observées dans certains cristaux de molécules cycliques [29], de telles interactions entre cycles aromatiques étant favorisées par la structure en doubles couches de Phe. De plus, des mesures de dichroïsme circulaire effectuées sur des films des énantiomères L ou D ont montré un signal dans les deux zones d'absorption, attestant que les deux types de molécules de Phe étaient chirales, la symétrie des signaux pour les deux énantiomères attestant la conservation de la chiralité lors du dépôt [10]. Enfin, des analyses de RMN du proton et de spectrométrie de masse couplée à la chromatographie sur colonne LC/MS ont été effectuées sur des 
solutions aqueuses obtenues par dissolution des films. Ces mesures montrent que les films, même ceux qui sont légèrement colorés parce qu'ils ont été déposés à hautes fluences ou parce qu'ils ont été irradiés par le rayonnement UV, sont bien constitués de la molécule de phénylalanine et ne comportent pas d'impuretés.

Au contraire, les films de Tyr, qui n'ont pas de structure ordonnée des cycles, ne donnent pas d'émission supplémentaire. Dans ce cas, la coloration jaune provient d'une dégradation partielle des molécules. En effet, la RMN et la LC/MS de films redissous, montrent la présence, en plus de celle des molécules de tyrosine, d'impuretés en faible concentration, non identifiées, dont la proportion augmente avec la fluence. La présence du groupe $\mathrm{OH}$ modifie donc notablement la stabilité de la molécule vis-à-vis de l'ablation.

\subsection{Films de calix-arènes}

Le tertio-butyl calix-[4]-arène est un macrocycle constitué de 4 cycles phénol reliés en ortho par un groupe méthylène et substitués en para par un groupe tertio butyl. La molécule a une conformation en cône à cause des fortes liaisons hydrogène reliant les groupes $\mathrm{OH}$. L'étude de cette molécule permet d'illustrer d'autres aspects de la préparation de films organiques par PLD, en particulier parce qu'il est possible de comparer les propriétés des films préparés par PLD à celles de films obtenus à partir de solutions ou par évaporation thermique ET.

Le comportement avec la fluence des propriétés structurales et optiques fait apparaitre trois zones de fluence. Le seuil d'ablation, déterminé par l'évolution avec la fluence de la profondeur ablatée par impulsion est particulièrement faible, $30 \mathrm{~mJ} / \mathrm{cm}^{2}$. A basse fluence, de 40 à $220 \mathrm{~mJ} / \mathrm{cm}^{2}$ environ, la conservation de la structure chimique et de la conformation en cône est assurée. A plus haute fluence, intervient une modification de la conformation et au-delà de $750 \mathrm{~mJ} / \mathrm{cm}^{2}$ la molécule est décomposée. Les films, amorphes, ont des indices de réfraction bien supérieurs à ceux de films préparés par ET (par exemple 1,62 au lieu de 1,56 à 632, $8 \mathrm{~nm}$ ). De plus, l'augmentation de la fluence permet une densification des films. La morphologie des films cristallisés obtenus par recuit ex-situ correspond à une échelle beaucoup plus fine (de l'ordre de $100 \mathrm{~nm}$ ) que celle des films déposés par ET et recuits (de l'ordre du micron), ce qui est beaucoup plus favorable au guidage optique.

\subsection{Films de protéines}

La possibilité de déposer des films de protéines sans dégradation de leur structure a été étudiée dans le cas de l'albumine sérique bovine, une protéine globulaire, comportant 583 acides aminés $(\mathrm{M}=66$, $4 \mathrm{kDa}$ ). Des films de quelques centaines de nm d'épaisseur ont été déposés dans une gamme de fluence s'étendant de40 à $1000 \mathrm{~mJ} / \mathrm{cm}^{2}$.

La conservation de la structure de la molécule a été vérifiée par spectroscopie IR (Figure 5). Les spectres, constitués des bandes caractéristiques des protéines, sont très semblables à celui de la poudre, sauf aux faibles fluences, pour lesquelles la présence de bandes additionnelles indique une décomposition partielle. Comme dans le cas de la phénylalanine, la structure chimique n'est préservée qu' au-dessus d'une fluence seuil de l'ordre de $80 \mathrm{~mJ} / \mathrm{cm}^{2}$ pour BSA.

L'élargissement vers les grands nombres d'onde de la bande Amide I, située vers $1654 \mathrm{~cm}^{-1}$ et la modification des intensités relatives du doublet de la bande Amide III située vers 1305 et $1250 \mathrm{~cm}^{-1}$ indiquent une légère modification de la structure secondaire de la protéine: la proportion de la conformation en hélice $\alpha$ a diminué au profit de celle en pelote statistique. De telles modifications de la structure secondaire ont été observées aussi bien pour des films de fibroine de la soie déposés par PLD à $351 \mathrm{~nm}$ [18] que pour des films de peroxidase du raifort (HRP) préparés par MAPLE [23]. Dans notre cas, la conservation de l'activité biologique reste à vérifier pour renforcer la pertinence de la PLD par rapport à la méthode MAPLE. 

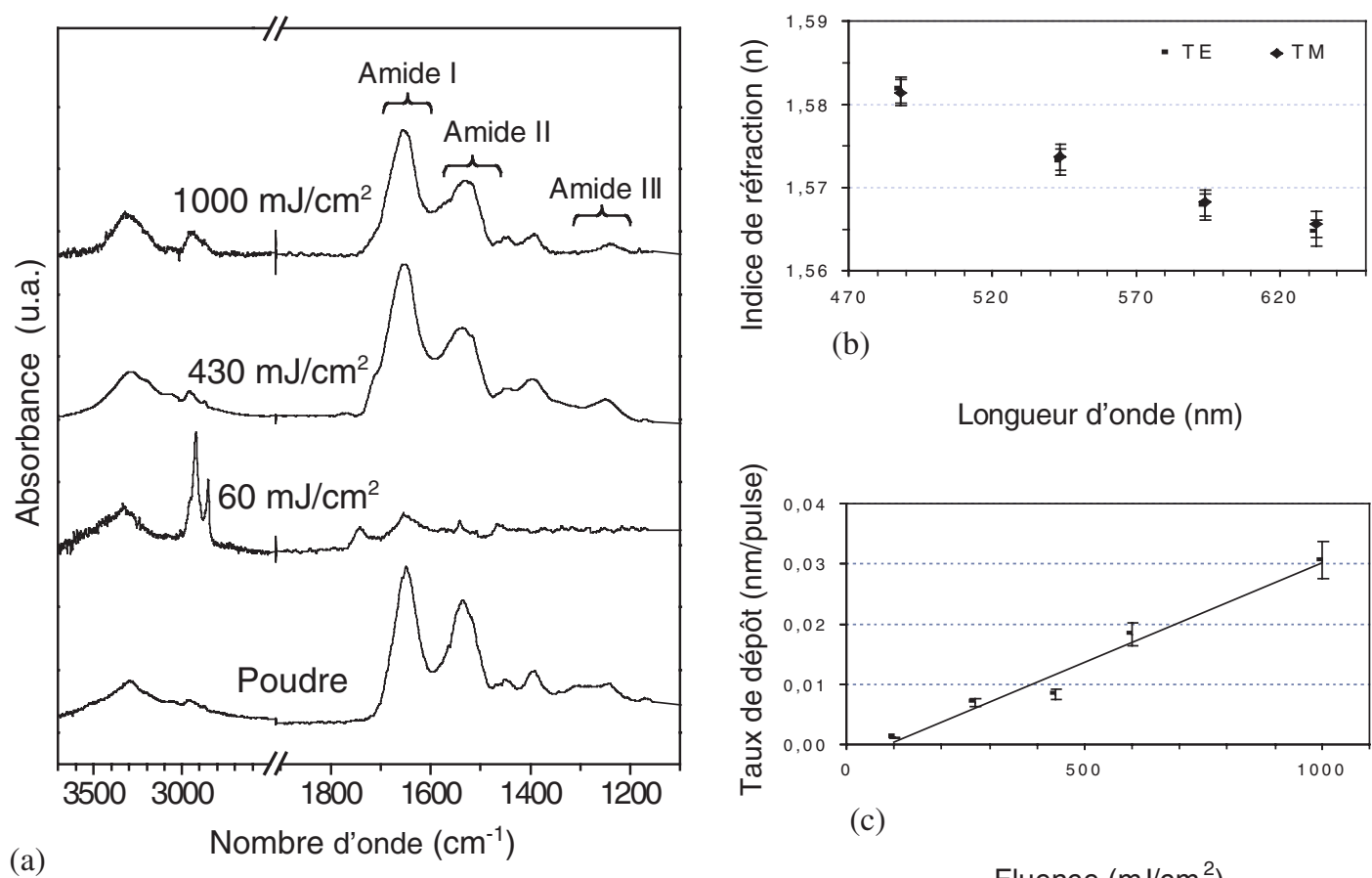

(b)

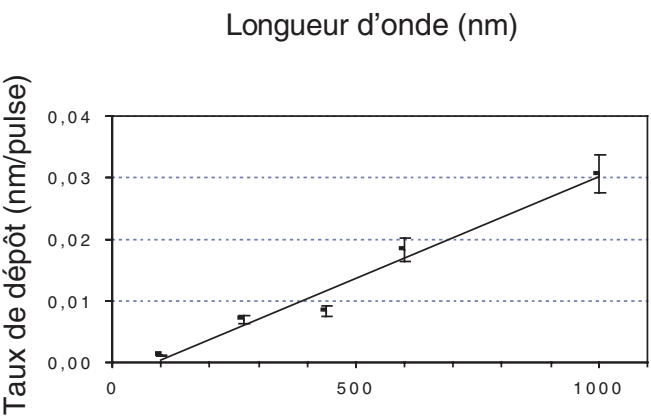

(c)

Fluence $\left(\mathrm{mJ} / \mathrm{cm}^{2}\right)$

Figure 5. (a) Spectre IR de la poudre et des films de BSA déposés à différentes fluences, (b) Courbe de dispersion de l'indice de réfraction d'un film de BSA déposé à $800 \mathrm{~mJ} / \mathrm{cm}^{2}$, (c) Evolution du taux de dépôt de BSA avec la fluence.

Les indices de réfraction des films de BSA, mesurés par m-lines à plusieurs longueurs d'onde, sont isotropes et leur courbe de dispersion a été déterminée (Figure 5b). Le taux de dépôt en fonction de la fluence (Figure 5c) permet de déterminer une fluence seuil de l'ordre de $80 \mathrm{~mJ} / \mathrm{cm}^{2}$, voisin du seuil de conservation de la molécule. Pour une même fluence, le taux de dépôt est environ un ordre de grandeur inférieur à celui de plus petites molécules comme la phénylalanine (par exemple respectivement 0,2 et $0,02 \mathrm{~nm} / \mathrm{impulsion}$ à $600 \mathrm{~mJ} / \mathrm{cm}^{2}$ )

\subsection{Morphologie de surface et guidage optique}

Notre avons également montré qu'il est possible, pour toutes les molécules étudiées, d'obtenir par PLD des films peu rugueux, qui ont des propriétés de guidage optique, ce qui ouvrent des perspectives pour des applications à des capteurs.

La morphologie de surface des films, analysée par AFM en mode non contact, dépend peu de la nature des molécules (Figure 6). La surface est constituée de particules arrondies de quelques centaines de $\mathrm{nm}$ de diamètre et quelques dizaines de nm de hauteur, ce qui conduit à des rugosités rms de 15 à $50 \mathrm{~nm}$ compatibles avec le guidage optique. Une propagation guidée sur une longueur de $2 \mathrm{~cm}$, limitée par la taille de l'échantillon, a été obtenue pour un film de Phe déposé à $80 \mathrm{~mJ} / \mathrm{cm}^{2}$ de rugosité rms de $22 \mathrm{~nm}$ (Figure 7). Le guidage optique est réduit à quelques $\mathrm{mm}$ si l'épaisseur ou la fluence de dépôt augmentent, à cause de l'augmentation de la rugosité. Pour les films de protéines la rugosité rms est particulièrement faible, ce qui a permis la première observation d'une propagation guidée dans un film de protéine. Il s'agissait d'un film de BSA de rugosité $\mathrm{rms}$ de $2 \mathrm{~nm}$, déposé à $270 \mathrm{~mJ} / \mathrm{cm}^{2}$, pour lequel une propagation guidée de quelques mm a été obtenue. Des rugosités rms beaucoup plus élevées, 


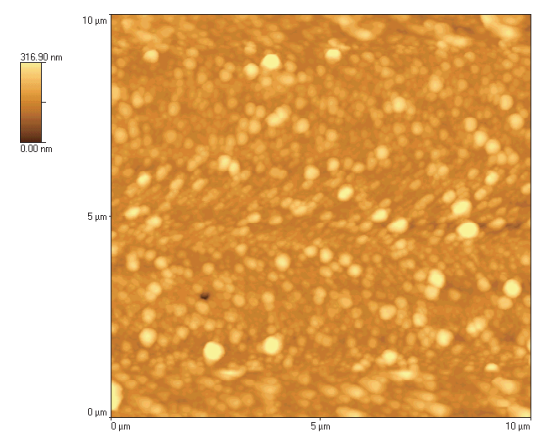

Figure 6. Image AFM $\left(10 \times 10 \mu \mathrm{m}^{2}\right)$ de la surface d'un film de Phe de $430 \mathrm{~nm}$ d'épaisseur déposé à $130 \mathrm{~mJ} / \mathrm{cm}^{2}$ sur $\mathrm{SiO}_{2}$ (rugosité rms $39 \mathrm{~nm}$, hauteur maximale $349 \mathrm{~nm}$ ).

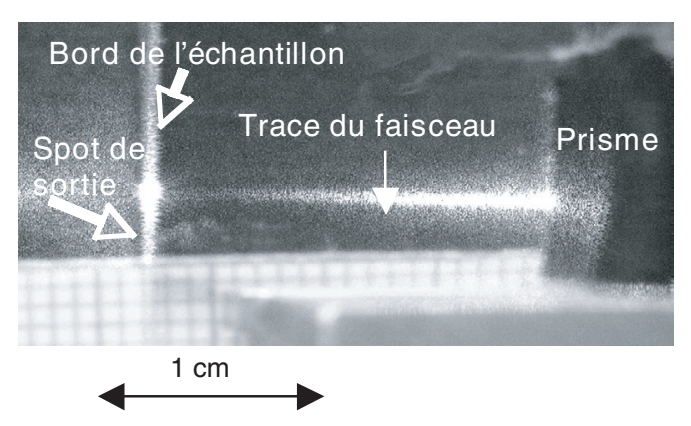

Figure 7. Propagation guidée dans un film de Phe de $320 \mathrm{~nm}$ d'épaisseur déposé sur du verre à $80 \mathrm{~mJ} / \mathrm{cm}^{2}$ de rugosité $\mathrm{rms} 22 \mathrm{~nm}$.

incompatibles avec des propriétés de guidage optique, ont été rapportées pour des films de protéines déposés par MAPLE, de l'ordre de 90 à $200 \mathrm{~nm}$ pour des films de HRP [23].

\section{CONCLUSION}

Avec l'exemple de deux acides aminés, phénylalanine et tyrosine, d'un calix-arène et d'une protéine globulaire, BSA, nous avons montré qu'il est possible de réaliser par PLD conventionnelle des films de molécules organiques sans dégradation chimique, pour des fluences de quelques dizaines à quelques centaines de $\mathrm{mJ} / \mathrm{cm}^{2}$. La PLD permet donc de transférer des molécules de différentes taille et complexité en conservant la composition chimique des molécules. De plus, la structure cristalline de Phe est également préservée. A plus petite échelle, la PLD peut induire une modification de l'arrangement moléculaire (désorganisation de l'hélice $\alpha$ de la protéine, renforcement des liaisons $\mathrm{H}$ de Phe, densification par la fluence de la tyrosine ou du calix-arène amorphe, changement de conformation du calix-arène ...). La rugosité de surface des films est suffisamment faible pour que le guidage optique ait été observé. L'existence d'une fluence seuil, en deçà de laquelle les molécules déposées sont partiellement dégradées, semble indiquer que deux régimes d'ablation se succèdent en fonction de la fluence. L'étude de la nature et de l'énergie des espèces présentes dans le panache, par spectroscopie optique et spectrométrie de masse, qui n'a encore été effectuée que dans les conditions, assez différentes, du MALDI, devrait permettre de comprendre les processus en jeu. Il en est de même pour les processus photochimiques en phase solide, susceptibles de modifier l'absorption des cibles et donc les conditions d'ablation. Enfin, une étude comparative effectuée sur des molécules identiques en utilisant le même laser serait du plus grand intérêt pour établir clairement les avantages et inconvénients de chacune des deux méthodes de dépôt par laser UV, dépôt par laser pulsé PLD conventionnel ou méthode MAPLE d'évaporation assistée par matrice.

\section{Références}

[1] Pulsed laser deposition of thin films, Ed: D. B. Chrisey, G. K. Hubler, (J. Wiley, New York, 1994).

[2] D. B. Chrisey, A. Piqué, R. A. McGill, J. S. Horwitz, B. R. Ringeisen, Chem. Rev. 103 (2003) 553.

[3] Organic light emitting devices: a survey, Ed: J. Shinar (Springer, Berlin, 2004).

[4] E. A. H. Hall Biosensors (Open University Press, Buckingham, 1990).

[5] W. W. Wang, G. K. Knopf, A. S. Bassi Proc. SPIT 5602 (2004) 41. 
[6] A. Ulman An introduction to ultrathin organic films: from Langmuir-Blodgett to self-assembly, (Academic Press, San Diego, 1991).

[7] T. Lippert, J. T. Dickinson Chem. Rev. 103 (2003) 453.

[8] R. Srinivarsan, B. Braren, D. E. Seeger, R. W. Dreyfus, Macromolecules 19 (1986) 916.

[9] Laser ablation of molecular substrates, Ed. S. Georgiou, F. Hillenkamp, Chem. Rev. 103 (2003) 317.

[10] M. A. Hernandez-Perez, Thèse de Doctorat, Université Lyon I, 2005.

[11] M. A. Hernandez-Perez, C. Garapon, C. Champeaux, J. C. Orlianges, J. Phys. Conf (à paraître).

[12] M. A. Hernandez-Perez, C. Garapon, C. Champeaux, A. W. Coleman, P. Shahgaldian, J. Mugnier, Appl. Phys. A 79 (2004) 1473.

[13] A. Hernandez-Perez, C. Garapon, C. Champeaux, P. Shahgaldian, A.W. Coleman, J. Mugnier, Appl. Surf. Sci. 208-209 (2003) 658.

[14] K. Itaka, T. Hayakawa, J. Yamaguchi, H. Koinuma, Appl. Phys. A 79 (2004) 875.

[15] G. B. Blanchet, C. R. Fincher, I. Malajovich, J. Appl. Phys. 94 (2003) 6181.

[16] X. Yang, Y. Tang, M. Yu, Q. Qin, Thin solid films 358 (2000)187.

[17] E. Ina, N. Matsumoto, E. Shikada, F. Kannari, Appl. Surf. Sci. 127-129 (1998) 574.

[18] Y. Tsuboi, M. Goto, A. Itaya, Chem. Lett. (1998) 521.

[19] Y. Tsuboi, N. Kimoto, M. Kabeshita, A. Itaya, J. Photochem. Photobiol. A 145 (2001) 209.

[20] Y. Tsuboi, M. Goto, A. Itaya, J. Appl. Phys. 89 (2001) 7917.

[21] A. Gutierrez-Llorente, R. Perez-Casero, B. Pajot, J. Roussel, R. M. Defourneau, D. Defourneau, J. L. Fave, E. Million, J. Perrière, Appl. Phys. A 77 (2003) 785.

[22] A. Gutierrez-Llorente, G. Horowitz, R. Perez-Casero, J. Perrière, J. L. Fave, A. Yassar, C. Sant, Organic Electronics 5 (2004) 29.

[23] B. R. Ringeisen, J. Callahan, P. K. Wu, A. Picqué, B. Spargo, A. A. McGill, M. Bucaro, H. Kim, D. M. Bubb, D. B. Chrisey, Langmuir 17 (2001) 3472.

[24] A. Piqué, P.Wu, B.R. Ringeisen, D.M. Bubb, J.S. Melinger, R.A. McGill, D.B. Chrisey, Appl. Surf. Sci. 186 (2002) 408.

[25] M. Jelinek, R. Cristescu, T. Kocourek, V. Vorlicek, J. Remsa, L. Stamatin, D. Mihailescu, D. B. Chrisey J. Phys. Conf (sous presse).

[26] K. Dreisewerd : Chem. Rev. 103 (2003) 395.

[27] L. V. Zhigilei, E. Leveugle, B. J. Garrison, Y. G. Yingling, M. I. Zeifman, Chem. Rev. 103 (2003) 321.

[28] N. Bityurin, B. S. Lukyanchuk, M. H. Hong, T. C. Chong, Chem. Rev. 103 (2003) 519.

[29] J. R. Lakowicz Principles of fluorescence spectroscopy (Kluwer Academic, New York, 1999). 